\title{
Schweizerische Gesellschaft für Rheumatologie (SGR)
}

Peter M. Villiger

Past-Präsident, Ordinarius für Rheumatologie und Klinische Immunologie, Direktor und Chefarzt, Inselspital Bern

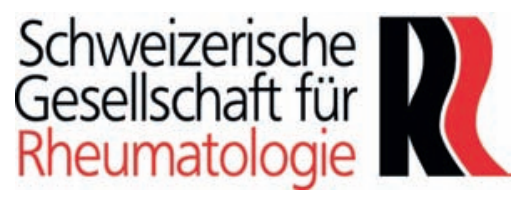

\section{Rheumatologie}

Die Rheumatologie befasst sich mit der Prävention, Diagnostik, nichtoperativen Therapie und Rehabilitation von Krankheiten des Bewegungsapparates. Hierzu gehören degenerative Erkrankungen der Gelenke und der Wirbelsäule; Weichteilerkrankungen; Knochen- und Stoffwechselkrankheiten/Osteoporose; schmerzhafte und funktionelle Störungen, die sich im Bewegungsapparat manifestieren.

Das Fachgebiet der Rheumatologie umfasst ferner die entzündlichen Erkrankungen des muskuloskelettalen Systems, des Bindegewebes und der Blutgefässe (systemische Autoimmunerkrankungen).

\section{Werdegang des Fachgebietes}

Die Rheumatologie im deutschsprachigen Raum hat ihre Wurzeln in der Bademedizin und befasste sich primär mit Erkrankungen des Bewegungsapparates. Demgegenüber beschäftigt sich die Rheumatologie in den angelsächsischen Ländern mit klinischer Immunologie, konkret mit den systemischen Autoimmunerkrankungen. In der Schweiz (wie auch in Frankreich) werden durch die Rheumatologie beide Bereiche abgedeckt.

Seit wenigen Jahren haben die Rheumatologen einen eigenen Facharzttitel, nachdem das Fachgebiet vorher als eine Subspezialität der Inneren Medizin oder der Physikalischen Medizin gelehrt wurde. Heute ist die SGR eine eigenständige Fachgesellschaft mit über 400 Mitgliedern.

\section{Partner}

Neben den ärztlichen Partnern (Innere Medizin, Physikalische Medizin, Rehabilitation, Orthopädische Chirurgie, Neurologie und Allergologie und Klinische Immunologie) arbeitet die SGR auch eng mit der Rheumaliga Schweiz zusammen. Diese widmet sich in erster Linie der Beratung des Laienpublikums. Sie versorgt Interessierte mit Informationsschriften und bietet verschiedenste
Kurse an. Kürzlich wurden neugestaltete Internetplattformen der SGR und der Rheumaliga Schweiz aufgeschaltet, die umfassende Informationen sowohl für Laien wie auch für Health Professionals und für Ärzte bereitstellen. Wir empfehlen ihnen den Besuch folgender Adressen:

- www.rheuma-schweiz.ch;

- www.rheuma-net.ch;

- www.rheumaliga.ch.

\section{Themen}

Zusammen mit der Rheumaliga Schweiz wurden fünf Hauptthemen gewählt, die mittels regionaler Veranstaltungen und Informationsbroschüren in den kommenden Jahren dem Publikum nähergebracht werden. Es handelt sich um:

- Osteoporose;

- Rückenschmerzen;

- Arthrose;

- Arthritis;

- Weichteilrheuma (z. B. Fibromyalgie).

Die genannten Schwerpunkte haben wesentliche gesundheitspolitische Bedeutung, da sie erhebliche direkte und indirekte Kosten verursachen.

\section{Von der Bademedizin zur Hightech-Disziplin}

Die Erkenntnisse betreffend Pathogenese entzündlich-rheumatischer Erkrankungen der vergangenen Jahre haben zu bahnbrechenden Neuerungen im therapeutischen Management geführt. Nach den Tumor-Nekrose-Faktor-(TNF-) Hemmern kamen kürzlich der B-Lymphozytendepletierende monoklonale Antikörper Rituximab und danach Abatacept, ein Hemmer der Kostimulation von Lymphozyten, auf den Markt. Diese gentechnologisch hergestellten Therapeutika werden dicht gefolgt von weiteren Molekülen, die der gezielten Regulation von Entzündungs- und Immunvorgängen dienen und in den kommenden Jahren das Armamentarium der Rheumatologen bereichern werden. Mit jedem neuen Biologikum werden die Differentialindikation wichtiger und das therapeutische Vorgehen komplexer. Für Herausforderung ist mit anderen Worten gesorgt. 